\title{
THREE-DIMENSIONAL LOCAL MHD SIMULATIONS OF HIGH STATES AND LOW STATES IN MAGNETIC ACCRETION DISKS
}

\author{
T. MATSUZAKI AND R. MATSUMOTO \\ Department of Physics, Faculty of Science, Chiba University, \\ 1-33 Yayoi-Cho, Inage-Ku, Chiba 263, Japan \\ T. TAJIMA \\ Institute for Fusion Studies, the University of Texas at Austin, \\ Austin, TX 78712, USA \\ AND \\ K. SHIBATA \\ National Astronomical Observatory, Mitaka, Tokyo 181, Japan
}

Black hole candidates sometimes show a transition between the high (or soft) state and the low (or hard) state. In the low state, low frequency time variations are much larger than the high state. A possible mechanism of the large-amplitude, sporadic time variabilities in the low-state is the magnetic energy release in low- $\beta\left(\beta=P_{g a s} / P_{\text {mag }}<1\right)$ disks (Mineshige, Kusunose $\&$ Matsumoto 1995). It had been thought that low- $\beta$ disks cannot exist because buoyant escape of magnetic flux due to the Parker instability may set the lower limit for $\beta$ inside the disk. Shibata, Tajima \& Matsumoto (1990), however, pointed out that in accretion disks, once a low- $\beta$ disk is formed, it can stay in low- $\beta$ state partly because the growth rate of the Parker instability decreases when $\beta<1$. They suggested that magnetic accretion disks fall into two types; high- $\beta$ disks and low- $\beta$ disks.

We carried out local three-dimensional magnetohydrodynamic (MHD) simulations of a gravitationally stratified, isothermal Keplerian disk initially threaded by azimuthal magnetic field. Since both differential rotation and vertical gravity are included, the magnetorotational (or Balbus \& Hawley) instability (Balbus \& Hawley 1991) couples with the Parker instability when $\beta \sim 1$. Local Cartesian coordinate is used with $x, y, z$ in the radial, azimuthal, and vertical direction, respectively. The vertical gravity is as- 

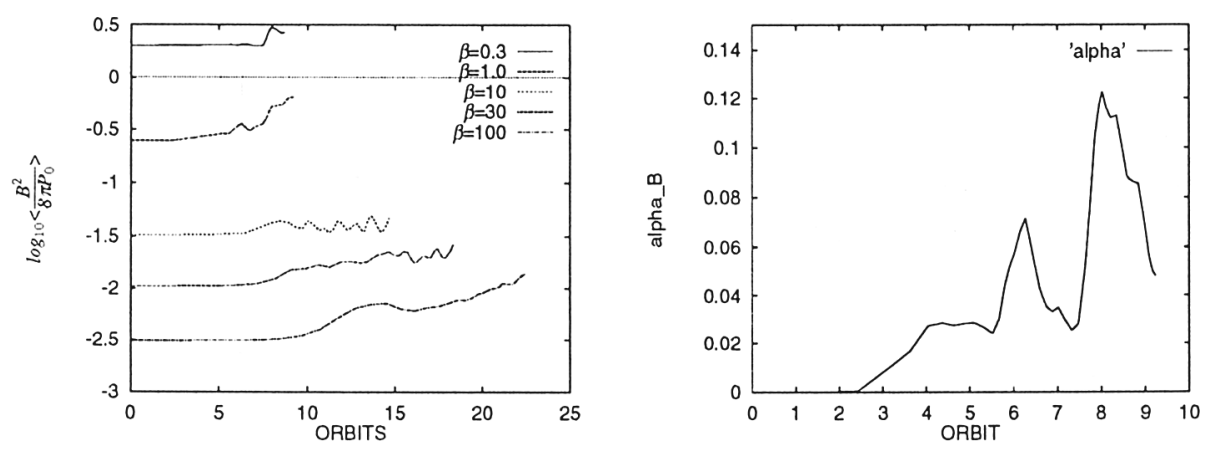

Figure 1. Time evolution of the mean magnetic field strength $\left\langle B^{2} /\left(8 \pi P_{0}(0)\right)\right\rangle$ for various initial $\beta$ (left panel) and the angular momentum transport rate $\alpha_{B}=-\left\langle B_{x} B_{y} /\left(4 \pi P_{0}(0)\right)\right\rangle$ for a model with $\beta=1$ (right panel). The unit of time is the rotation time.

sumed to be $g_{z}=-G M z /\left(r_{0}^{2}+z^{2}\right)^{3 / 2}$ where $r_{0}$ is the radius from the gravitating center. We assume that $\beta$ is uniform at the initial state. The size of the simulation box is $\left(L_{x}, L_{y}, L_{z}\right)=(1 H, 18 H, 16 H)$, where $H$ is the scale height defined by using the sound speed $C_{s}$ and Keplerian angular speed $\Omega_{K}$ as $H=C_{s} / \Omega_{K}$. The azimuthal boundaries are periodic. The radial boundaries are treated by using the sliding periodic condition.

The left panel of Figure 1 shows the time evolution of the mean magnetic field strength $\left\langle B^{2} /\left(8 \pi P_{0}(0)\right)\right\rangle$ for various initial plasma $\beta$, where $P_{0}(0)$ is the initial equatorial pressure. Numerical results indicate that in high- $\beta$ disks, the amplification of magnetic fields due to the Balbus-Hawley instability saturates when $\beta \sim 10-30$. The disk approaches to a gas pressure dominated, quasi-steady state. The effective value of the viscosity parameter is $\alpha_{B}=-\left\langle B_{x} B_{y} /\left(4 \pi P_{0}(0)\right)\right\rangle \sim 0.01$. These results are consistent with those reported by Stone et al. (1996). When the initial magnetic energy is comparable to the thermal energy $(\beta \sim 1)$, however, the disk stays in the low- $\beta$ state for time scale longer than the rotation period. In such disks, the amplification of magnetic fields due to the coupling of the Balbus \& Hawley instability and the Parker instability overcomes the buoyant loss of magnetic flux. The effective magnetic viscosity in low- $\beta$ state is the order of 0.1 as shown in the right panel of Figure 1. When the magnetic energy stored in the low- $\beta$ disk is released, we expect large amplitude sporadic time variations as observed in low-state disks.

\section{References}

Balbus, S. A., \& Hawley, J. F. (1991), ApJ, 376, 214.

Mineshige, S., Kusunose, M., \& Matsumoto, R. (1995), ApJ, 445, L43.

Shibata, K., Tajima, T., \& Matsumoto, R. (1990), ApJ, 350, 295.

Stone, J. M., Hawley, J. F., Gammie, C. F., \& Balbus, S. A. (1996), ApJ, 463, 656. 